\title{
Ventriculitis: A Rare Case of Primary Cerebral Toxoplasmosis in AIDS Patient and Literature Review
}

\author{
Gláucia Fernandes Cota ${ }^{1}$, Elisa Caroline Pereira Assad ${ }^{1}$, Paulo Pereira Christo ${ }^{1}$, Alexandre Varella Giannetti ${ }^{2}$, José Augusto \\ Malheiros dos Santos Filho' ${ }^{2}$, and Marcelo Antonio Pascoal Xavier ${ }^{3}$ \\ ${ }^{1}$ Service of Infectious Diseases of Eduardo de Menezes Hospital; ${ }^{2}$ Federal University of Minas Gerais, Department of Neurosurgery; ${ }^{3}$ Federal \\ University of Minas Gerais, Department of Pathologic Anatomy; Belo Horizonte, MG, Brazil
}

\begin{abstract}
Cerebral toxoplasmosis remains the most important neurological opportunistic infection and the most common cause of intracerebral mass lesion in patients with acquired immunodeficiency syndrome (AIDS). We report a case of an adult AIDS patient with an atypical pattern of toxoplasma encephalitis, presenting with ventriculitis and obstructive hydrocephalus without any focal parenchymal lesion.
\end{abstract}

Key-Words: Cerebral toxoplasmosis, ventriculitis, hydrocephalus, AIDS.

Toxoplasma encephalitis (TE) is the most frequent cause of focal central nervous system (CNS) infection complicating the acquired immunodeficiency syndrome [1]. If untreated, TE is fatal. TE remains a highly prevalent disorder of the central nervous system, even in the late highly active antiretroviral therapy (HAART) era, particularly among severely immunosuppressed patients and in absence of prophylaxis [2].

The radiological classical pictures of TE are multiple parenchymal lesions, with ring enhancement, which are generally localized at the corticomedullar junction, in the white matter, or the basal ganglia [3]. Other forms of CNS involvement are rare. In cases of severe immunosuppression, the neuroimages can be completely atypical, misleading radiologists and clinicians [4]. In this report we described an unusual presentation of cerebral toxoplasmosis in an adult AIDS patient in whom ventriculitis and obstructive hydrocephalus without evidence of focal parenchymal lesion were observed as the primary manifestations of cerebral toxoplasmosis.

\section{Case Report}

A 24-year-old woman with no significant past medical history, was admitted to Eduardo de Menezes Hospital (Belo Horizonte, Brazil) with a history of headache and vomiting for 15 days. Three days before admission, the patient developed generalized seizure followed by altered state of consciousness. The patient was previously evaluated at another institution where a rapid human immunodeficiency virus (HIV) testing was performed with a positive result, and referred to our hospital. The general physical examination was normal. The neurological examination revealed drowsiness but no focal deficits, cranial nerve palsies or meningism. The fundoscopic examination was normal. After admission, the level of

Received on 10 November 2007; revised 15 January 2008. Address for correspondence: Dr. Gláucia Fernandes Cota. Av. Dr. Cristiano Rezende, 2213, Bairro Bonsucesso, Belo Horizonte, Minas Gerais, Brazil. Zip code: 30.622-020. Phone: 0xx31-3383-8000. E-mail: gcota@ufmg.br.

The Brazilian Journal of Infectious Diseases 2008;12(1):101-104. (C) 2008 by The Brazilian Journal of Infectious Diseases and Contexto Publishing. All rights reserved. consciousness worsened and the patient remained four days in mechanical ventilation and seven days in an intensive care unit. Her clinical condition improved although drowsiness, mental confusion, and mild cognitive deficit were present.

The contrast-enhanced cranial computed tomography (CT) scan showed enlargement of lateral ventricles with enhancement of the ventricular surface (ependymitis), mainly on the left. CT scan also demonstrated periventricular hypodensities, suggesting interstitial edema and a slight hypodensity of the left lateral ventricle cerebral fluid (CSF). There was no evidence of focal parenchymal lesion (Figures $1 \mathrm{~A}$ and $1 \mathrm{~B})$. Because we could not absolutely exclude the possibility of intracerebral mass lesion on CT, empirical treatment for TE was initiated with pyrimethamine, sulfadiazine, and folinic acid at admission.

The HIV-1 enzyme-linked immunosorbent assay (ELISA) and Western Blot were positive. CD4 ${ }^{+} \mathrm{T}$ lymphocyte count was 65 cells $/ \mathrm{mm}^{3}$. Chest radiography was normal. Serum cytomegalovirus (CMV) IgG and Toxoplasma $\mathrm{IgG}$ were positive, consistent with previous exposure.

A lumbar puncture was performed although there was relative contraindication to the procedure. The CSF analysis showed $1 \mathrm{cell} / \mathrm{mm}^{3}$ lymphocytes, a protein level of $1,159 \mathrm{mg} /$ $\mathrm{dL}$, and a glucose concentration of $33 \mathrm{mg} / \mathrm{dL}$ (serum level was $94 \mathrm{mg} / \mathrm{dL}$ ). The CSF Gram stain, India ink, cryptococcal antigen and cultures for bacteria, mycobacterium and fungal were negative. Blood and CSF examination were negative for syphilis. The CSF polymerase chain reaction (PCR) for cytomegalovirus (CMV) was also negative. The results of the CSF analysis were believed to be consistent with tuberculous meningitis. Therefore, the patient was treated with antituberculosis drugs, including isoniazid, rifampin and pyrazinamide plus prednisone. Specific therapy for toxoplasmosis was maintained.

Three weeks after starting therapy for toxoplasmosis, magnetic resonance imaging (MRI) demonstrated lateral ventricles enlargement without any evidence of focal brain lesion. The flair and T2-weighted images showed extensive periventricular white matter hyperintensity, likely representing edema (Figures 1C and 1D). The postcontrast T1-weighted image disclosed ependymal enhancement particularly around the left ventricle, whose CSF was less hypointense than usual. 
Figure 1. $A$ and B (Post-contrast CT scan): one notes the enlargement of the lateral ventricles, and ependymal enhancement. $C$ (Flair MRI) and D (T2 weighted MRI): theses studies disclose the hyperintensity of the periventricular white mater, evoking edema related to the inflammatory process and transudation of ventricle cerebral fluid (CSF) secondary to the ventricular blockage. E and F (Post-contrast T1 weighted MRI): it can be observed the ventricular dilatation, secondary to obstruction of both foramina of Monro. There is enhancement of the ependymal layer and the left ventricle CSF is less hypodense than usual, suggesting a higher protein content or presence of debris

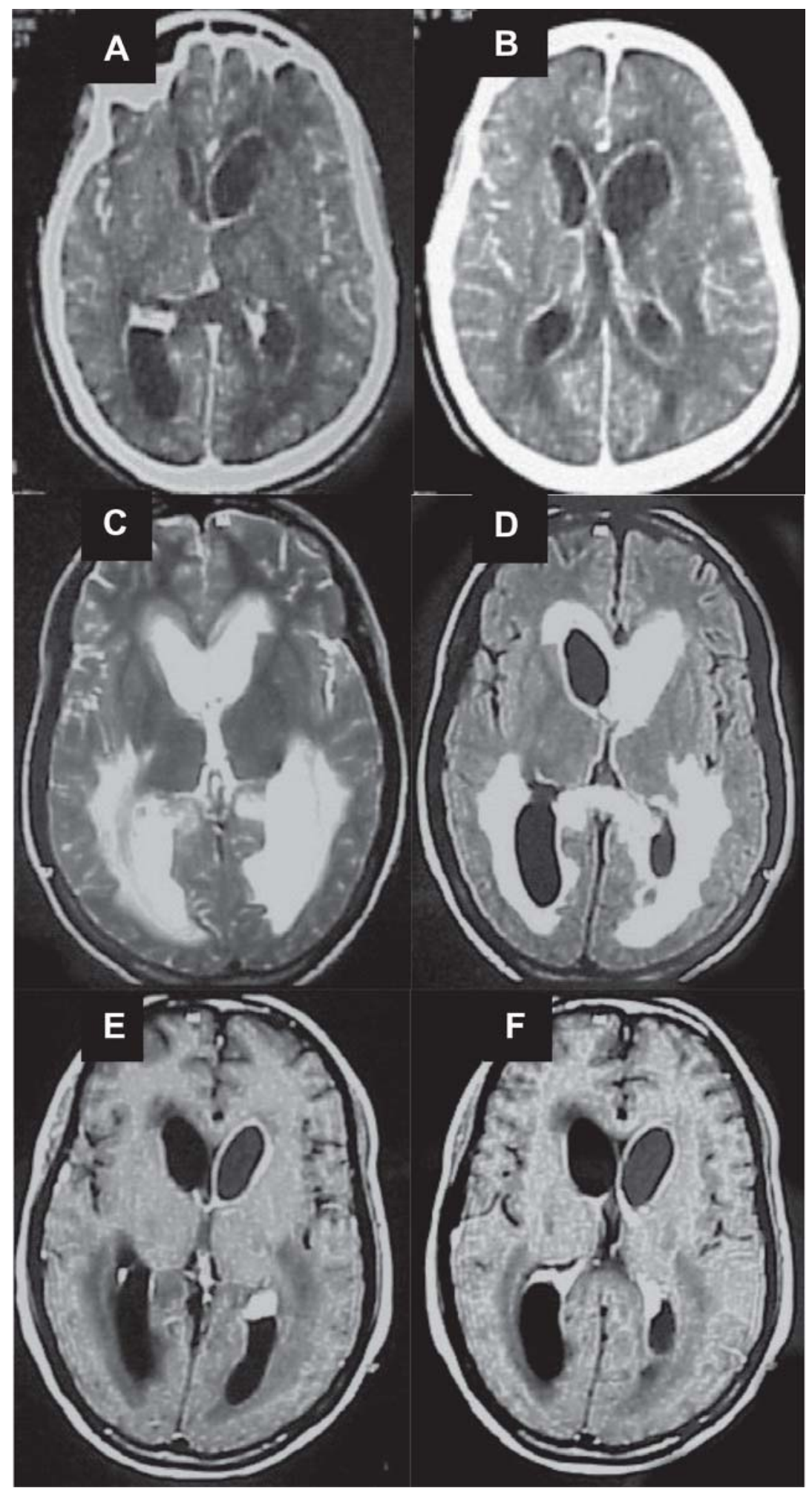

www.bjid.com.br 
Figure 2. In hematoxilin \& eosin coloration; 40x, can be observed: A) Diffuse infiltrated with lymphocytes, macrophages and plasma cells. B) Microglial nodule. C) Lymphocytic perivascular infiltration and hyalinization, thickening of the arteriolar wall.

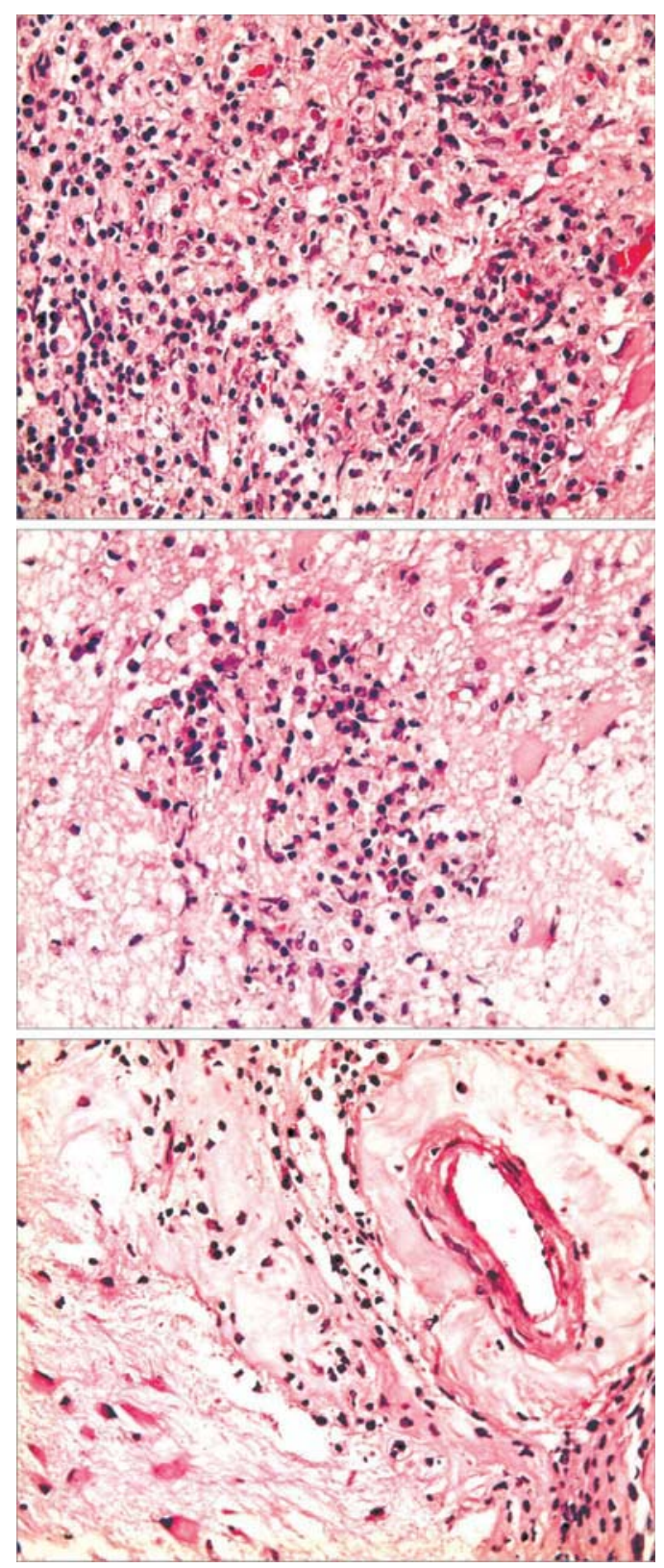

These findings are consistent with ventriculitis (Figures 1E and $1 \mathrm{~F}$ ).

Six weeks after admission, a neurosurgical procedure was done. Under general anesthesia, a rigid endoscope (Karl Storz, Germany) was introduced into the right lateral ventricle through a right parasagital frontal bore hole. A septostomy was performed allowing access to the left lateral ventricle, which presented a muddy CSF. Some white spot lesions were present at the ventricular walls. Using fine forceps, five biopsies were taken. Both foramina of Monro were obstructed. The right one was opened using the monopolar as a stiletto, permitting penetration to the third ventricle. A standard endoscopic third ventriculostomy was done. After access to the cisterns, it could be realized that there was no subarachnoid abnormality or inflammation, therefore antituberculosis drugs were discontinued. A ventricular catheter was left to maintain the apertures performed and keep the communication between the lateral and third ventricles, to the prepontine cistern.

Neuropathological examination revealed inflammatory tissue infiltrated with lymphocytes, macrophages and plasma cells, areas with microglial nodules and lymphocytic perivascular infiltration and hyalinization, thickening of arteriolar wall (Figure 2). This pattern is highly suggestive of toxoplasmosis infection. Structures compatible with Toxoplasma gondii were also observed in hematoxilin-eosin coloration (HE).

Antiretroviral therapy was initiated with zidovudine, lamivudine, and efavirenz. A follow-up CT scan performed after seven weeks of therapy for toxoplasmosis showed significant improvement of hydrocephalus and periventricular hypodensities. Acute therapy for toxoplasmosis was maintained for eight weeks followed by secondary prophylaxis. Her neurological condition improved progressively.

\section{Discussion}

Cerebral toxoplasmosis in AIDS patients typically presents on neuroimaging studies as multiple lesions, but a solitary mass may account for nearly one third of the patients [3]. TE lesions are more often located at the basal ganglia, although any portion of the brain may be involved, and appear as ringenhancing lesions [3]. Nonenhancing abnormalities on CT scan have been reported in $6 \%$ to $20 \%$ of the cases [5]. Furthermore, if the CT scan has yielded unremarkable findings during the initial screening, MRI is recommended because it is more sensitive and will detect small additional lesions in some cases [5]. There are occasionally unusual appearances of CNS toxoplasmosis that make diagnosis by standard imaging techniques difficult or impossible. Although ventriculitis and consecutive obstructive hydrocephalus are characteristic findings in congenital toxoplasmosis [6], they are rarely seen in adult immunocompromised hosts.

To our knowledge, cerebral toxoplasmic ventriculitis has only been reported in eight adults AIDS patients [7-13] and in two immunosuppressed non-AIDS patients $[14,15]$. In the AIDS patients, toxoplasmic ventriculitis was accompanied by hydrocephalus in seven cases [7-10,12,13] and occurred as the primary manifestations of toxoplasmosis in five [8$10,12,13]$. In the other three patients it occurred as complications of a preexistent, recognized cerebral toxoplasmosis [7,11].

In addition, hydrocephalus without mass lesion is an unusual presentation of TE and was the only abnormal finding on CT scan in an adult AIDS patient reported by Nolla Sals et 
al. [16]. In that case, the neuropathological examination revealed hydrocephalus secondary to aqueduct stenosis caused by multiple necrotic foci in the mesencephalon and no signs of ventriculitis were present.

It is important to recognize that subacute periventricular encephalitis can occur in opportunistic toxoplasmosis, in order to assure timely treatment. If left untreated or if diagnosis is delayed, TE is often lethal. In all cases reported of toxoplasmic ventriculitis, the patients died $[7,15]$, and in four cases specific therapy for toxoplasmosis was considered $[7,9,14]$. Differing from those cases, our patient had a successful clinical course. It was possibly due to the early beginning of specific therapy for toxoplasmosis and surgical intervention.

In our case, ventriculitis accompanied by hydrocephalus were the only findings of cerebral toxoplasmosis. There was no evidence of focal parenchymal lesion. As we could not exclude the possibility of intracerebral mass lesion on the CT scan, empirical antitoxoplasmosis treatment was promptly started. Hydrocephalus was caused by blockage of the CSF flow within the ventricular system due to ependymitis with exudation of inflammatory material. In our patient, the examination of the CSF and blood were negative for other common opportunistic infections and her clinical condition without signs of deterioration offered further evidence of the possibility of cerebral toxoplasmosis.

The radiological differential diagnosis of periventricular enhancement in HIV infection is usually tuberculosis, lymphoma and viral ependymitis (CMV or varicela-zoster virus). The picture of enhancement can help distinguish the etiology. The presence of thin linear enhancement suggests a viral etiology whereas the presence of nodular enhancement suggests CNS lymphoma. The presence of band enhancement is less specific and can be seen with viral, lymphomatous and even tuberculous involvement [17]. Pyogenic ventriculitis should also be considered as a differential diagnosis. It is an uncommon but very severe complication of intracranial infection in adults requiring rapid diagnosis and therapy because of its high mortality. Irregular intraventricular debris seems to be the most specific abnormality on MRI, which usually can also demonstrate a periventricular high signal, ependymal contrast enhancement and hydrocephalus [18].

Our case stresses the importance that in HIV disease, neuroradiological and laboratory investigations can be grossly misleading and that cerebral toxoplasmosis should be considered in immunocompromised patients who present ventriculitis even if neuroimaging does not reveal any mass lesion.
The endoscopic biopsy seems to be useful to settle the diagnosis. The simultaneous treatment of the ventricular dilatation was important to accomplish the good result.

\section{References}

1. Luft B.J., Remington J.S. Toxoplasmic encephalitis in AIDS. Clin Infect Dis 1992;15(2):211-22.

2. Antinori A., Larussa D., Cingolani A., et al. Prevalence, associated factors, and prognostic determinants of AIDS-related toxoplasmic encephalitis in the era of advanced highly active antiretroviral therapy. Clin Infect Dis 2004;39(11):1681-91.

3. Ramsey R.G., Gean A.D. Neuroimaging of AIDS. I. Central nervous system toxoplasmosis. Neuroimag Clin N Am 1997;7(2):171-86.

4. Kastrup O., Wanke I., Maschke M. Neuroimaging of infections. NeuroRx 2005;2(2):324-32.

5. Ciricillo S.F., Rosenblum M.L. Use of CT and MR imaging to distinguish intracranial lesions and to define the need for biopsy in AIDS patients. J Neurosurg 1990;73(5):720-4.

6. Frenkel J.K. Pathology and pathogenesis of congenital toxoplasmosis. Bull NY Acad Med 1974;50(2):182-91.

7. Artigas J., Grosse G., Niedobitek F., et al. Severe toxoplasmic ventriculomeningoencephalomyelitis in two AIDS patients following treatment of cerebral toxoplasmic granuloma. Clin Neuropathol 1994;13(3):120-6.

8. Bourgouin P.M., Melançon D., Carpenter S., et al. Hydrocephalus and prominence of the choroid plexus: an unusual computed tomographic presentation of cerebral toxoplasmosis in AIDS. Can Assoc Radiol J 1992;43(1):55-9.

9. Cohen W., Koslow M. An unusual CT presentation of cerebral toxoplasmosis. J Comput Assist Tomogr 1985;9(2):384-6.

10. Eggers C., Vortmeyer A., Emskotter T. Cerebral toxoplasmosis in a patient with the acquired immunodeficiency syndrome presenting as obstructive hydrocephalus. Clin Neuropathol 1995; $14(1): 51-4$.

11. Sánchez J.F., Olmedo M.C., Pascua F.J., Casado I. Diabetes insipidus as a manifestation of cerebral toxoplasmosis in an AIDS patient. Rev Neurol 2000;30(10):939-40.

12. de Silva T., Raychaudhuri M., Poulton M., et al. Ventriculitis and hydrocephalus: an unusual presentation of toxoplasmosis in an adult with human immunodeficiency virus. J Neurol Neurosurg Psychiatry 2005;76(8):1074.

13. Sell M., Klingebiel R., Di Iorio G., Sampaolo S. Primary cerebral toxoplasmosis: a rare case of ventriculitis and hydrocephalus in AIDS. Clin Neuropathol 2005;24(3):106-11.

14. Best T., Finlayson M. Two forms of encephalitis in opportunistic toxoplasmosis. Arch Pathol Lab Med 1979;103(13):693-6.

15. Wongmongkolrit T., McPherson S.L., El-Naggar A., et al. Acute fulminant toxoplasma meningoencephalitis in a homosexual man. Acta Neuropathol (Berl) 1983; 60(3-4): 305- 8.

16. Nolla-Salas J., Ricart C., D’Olhaberriague L., et al. Hydrocephalus: an unusual CT presentation of cerebral toxoplasmosis in a patient with acquired immunodeficiency syndrome. Eur Neurol 1987;27(2): 130-2.

17. Guerini H., Helie O., Leveque C., et al. Diagnosis of periventricular ependymal enhancement in MRI in adults. J Neuroradiol 2003;30(1):46-56.

18. Fukui M.B., Williams R.L., Mudigonda S. CT and MR imaging features of pyogenic ventriculitis. AJNR Am J Neuroradiol 2001;22(8):1510-6. 